\title{
Un método para determinar un grupo de observaciones influyentes en la $S C E$ al ajustar modelos de rango completo
}

\author{
Implementation of a Method to Determine a Group of Influential \\ Observations in the $S C E$ when fitting Full Rank Models
}

Luis Francisco Rincón Suárę

franciscorincon@usantotomas.edu.co

\section{Resumen}

En este artículo se presenta un método que permite establecer la existencia de un grupo de observaciones influyentes para la $S C E$ al ajustar un modelo lineal de rango completo. En el análisis de residuales se utiliza la estadística $Q_{i}$ y el criterio se construye usando la distribución de las estadísticas mencionadas bajo el supuesto clásico $e_{i} \sim N\left(0 ; \sigma^{2}\right)$ y $\operatorname{Cov}\left(e_{i}, e_{j}\right)=0$ si $\left.i \neq j\right)$.

Palabras clave: modelo de rango completo, observaciones influyentes, parámetros estimados, suma de cuadrados residual.

\begin{abstract}
Throughout this paper is presented a method that allows us to identify the presence of an influential group of observations for the RSS after fitting a linear model of full rank. Over the residual analysis a $Q_{i}$ statistic is used and the criteria is built, using the distribution of the statistic mentioned under the assumption than $e_{i} \sim N\left(0 ; \sigma^{2}\right)$ and $\operatorname{Cov}\left(e_{i}, e_{j}\right)=0$ if $(i \neq j)$.
\end{abstract}

Key words: Estimated Parameters, Full rank Model, Influential Observations, Residual Sum of Squares.

\section{La estadística $Q_{i}$}

En la revista Comunicaciones en Estadística de la Universidad Santo Tomás, volumen 2 número 2 página 139, se expone la metodología para calcular la estadística

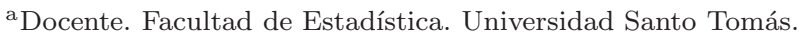


$Q_{i}$ que evaluada para el $i$-ésimo registro, mide el cambio en la $S C E$ cuando el modelo $Y=X \beta+e$ se ajusta después de eliminar este registro. Dicha estadística se calcula con la expresión

$$
Q_{i}=\frac{e_{i}^{2}}{1-h_{i i}}=S C E-S C E(i)
$$

donde $h_{i i}=X_{i}\left(X^{\prime} X\right)^{-1} X_{i}^{\prime}, S C E$ es la suma de cuadrados residual cuando el modelo se ajusta con los $n$ registros y $S C E(i)$ es la suma de cuadrados residual cuando el modelo se ajusta sin el $i$-ésimo registro.

Con el supuesto clásico $e_{i} \sim N\left(0 ; \sigma^{2}\right)$ y $\operatorname{Cov}\left(e_{i}, e_{j}\right)=0$ para $i \neq j$ se deduce $s^{2}$ el estimador insesgado de $\sigma^{2}$ la varianza residual del modelo original:

$$
\frac{e_{i}}{\sigma} \sim N(0,1) \quad \text { y } \quad \frac{(n-p) s^{2}}{\sigma^{2}} \sim \chi_{n-p}^{2}
$$

Se asocia a la estadística $T_{i}=\frac{\sqrt{Q_{i}\left(1-h_{i i}\right)}}{s}$ una distribución $T_{(n-p)}$ que permite establecer el siguiente criterio para la clasificación de observaciones influyentes. La $i$-ésima observación es influyente para la $S C E$ al ajustar el modelo $Y=X \beta+e$ si $\left|T_{i}\right| \geq t_{\alpha / 2}$

\section{El método propuesto}

Considerando que la estadística

$$
Q_{i}=S C E-S C E(i)
$$

evaluada para el $i$-ésimo registro, mide el cambio en la $S C E$ cuando el modelo $Y=X \beta+e$ se ajusta después de eliminar este registro, se define:

- La estadística

$$
Q_{(i, j)}=S C E(i)-S C E(i, j)=S C E-S C E(j \mid i)
$$

que mide el cambio en la $S C E$ cuando el modelo se ajusta después de eliminar la pareja de registros $(i, j), S C E(i, j)$ es la suma de cuadrados residual al ajustar el modelo después de eliminar la pareja de registros $(i, j)$ y $S C E(j \mid i)$ es la suma de cuadrados residual antes de ajustar el modelo, para ello se elimina el $j$-ésimo registro después de eliminar el registro $i$.

- La estadística

$$
Q_{(j \mid i)}=S C E(i)-S C E-S C E(j \mid i)
$$

que mide el cambio en la $S C E$ cuando antes de ajustar el modelo se elimina el $j$-ésimo registro después de eliminar el registro $i$. 
De lo anterior se deduce

$$
Q_{(i, j)}=S C E-S C E(i)+Q_{(j \mid i)}
$$

es decir

$$
Q_{(i, j)}=Q_{i}+Q_{(j \mid i)}
$$

lo cual significa que $Q_{(i, j)}$, es el impacto sobre la suma de cuadrados residual $S C E$ al eliminar la pareja de registros $(i, j)$, se puede calcular como la suma de $Q_{i}$, el impacto de eliminar el $i$-ésimo registro y el impacto $Q_{(j \mid i)}$, calculado al eliminar el registro $j$ después de eliminar el registro $i$. En consecuencia, $Q_{(i, j)}$ toma su valor máximo cuando $Q_{i}$ y $Q_{(j \mid i)}$ son valores máximos. Para determinar la pareja de observaciones $(i, j)$ que mayor impacto genera sobre $S C E$ se sugiere el siguiente procedimiento:

- Para los $n$ registros tomados en el análisis, estime el modelo $Y=X \beta+e$ y calcule la estadística $Q_{i}$ para $i=1,2, \cdots n$.

- Elimine la observación para la cual $Q_{i}$ toma el valor máximo, ajuste el modelo y calcule nuevamente la estadística $Q_{(j \mid i)}$ para las $n-1$ observaciones presentes.

- Cuando el procedimiento descrito se generaliza aplicando en cada paso el criterio de influencia establecido con la estadística $T_{i}$ para un nivel de significancia $\alpha$ y se detiene cuando $\left|T_{i}\right|<t_{\alpha / 2}$, como resultado se obtiene el grupo de observaciones que mayor impacto generan en la $S C E$ al ajustar el modelo.

\section{Ejemplo}

Se ilustra el desarrollo teórico expuesto en las secciones anteriores con el siguiente ejemplo aplicado a datos simulados para el modelo $Y=\beta_{0}+\beta_{1} X_{1}+\beta_{2} X_{2}+e$. Para los datos simulados, inicialmente se ajusta el modelo con las 20 observaciones, se ilustra la salida típica calculada en SAS, con la prueba de normalidad de los residuales. Para $i=1,2, \ldots 20$, se calculan las estadísticas $Q_{i}, T_{i}$ y el $\mathrm{P}$-valor de $T_{i}$, además se muestra la variación porcentual de la suma de cuadrados residual $S C E$ resultante de eliminar el 1-ésimo registro. En este paso y utilizando como criterio de influencia el $\mathrm{P}$-valor de $T_{i}$, se caracteriza la observación más influyente para la $S C E$. Se elimina esta observación, se ajusta nuevamente el modelo y se describen los cambios ocurridos en la $S C E$ y en la prueba de normalidad de los residuales, luego se repite el proceso hasta que el criterio determine que ya no hay observaciones influyentes. 
- Ajuste inicial del modelo con las 20 observaciones.

$\begin{array}{cccc}\text { Obs } & \text { Y } & \text { X1 } & \text { X2 } \\ 1 & 38.91 & 4.2 & 1.9 \\ 2 & 25.57 & 1.2 & 3.1 \\ 3 & 26.71 & 1.4 & 2.8 \\ 4 & 26.67 & 1.3 & 2.9 \\ 5 & 23.94 & 1.8 & 2.2 \\ 6 & 33.43 & 1.5 & 3.0 \\ 7 & 26.07 & 2.0 & 1.9 \\ 8 & 25.13 & 2.2 & 1.6 \\ 9 & 24.32 & 2.4 & 1.3 \\ 10 & 26.24 & 2.6 & 1.0 \\ 11 & 25.27 & 2.5 & 1.3 \\ 12 & 32.76 & 3.3 & 2.3 \\ 13 & 28.81 & 3.0 & 1.7 \\ 14 & 25.35 & 3.2 & 1.5 \\ 15 & 26.93 & 3.4 & 2.1 \\ 16 & 28.80 & 3.8 & 2.6 \\ 17 & 29.74 & 4.0 & 1.8 \\ 18 & 29.62 & 4.5 & 2.3 \\ 19 & 30.28 & 4.6 & 1.5 \\ 20 & 34.78 & 4.8 & 3.2\end{array}$

La siguiente es la salida del ajuste del modelo

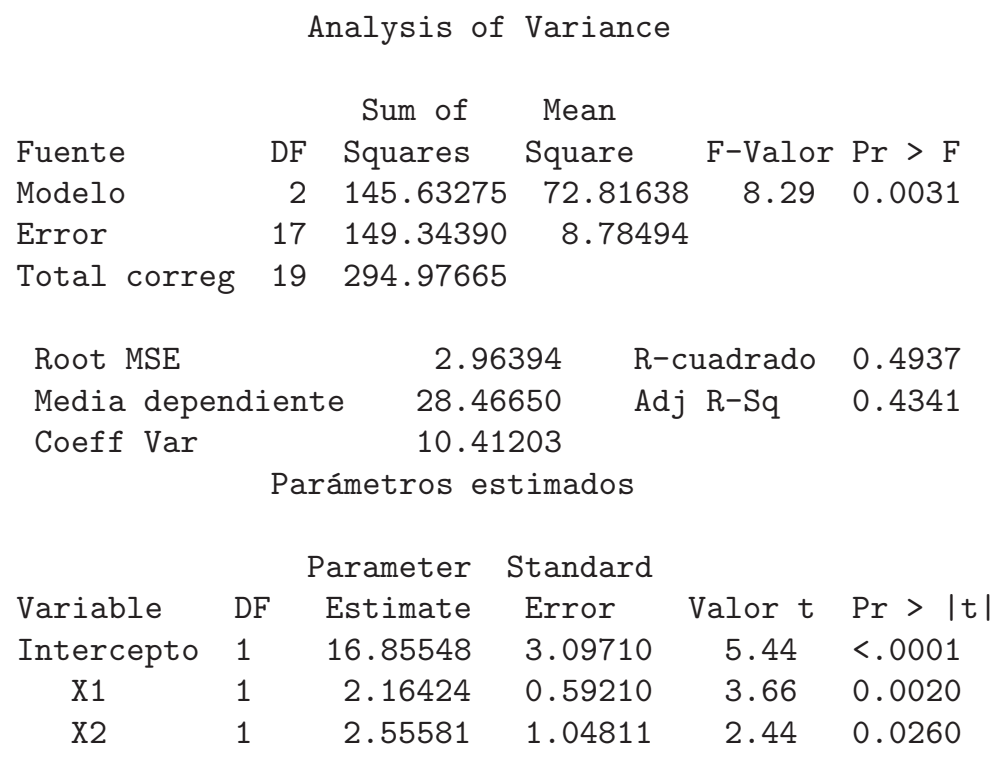

Comunicaciones en Estadística, diciembre 2010, Vol. 3, No. 2 


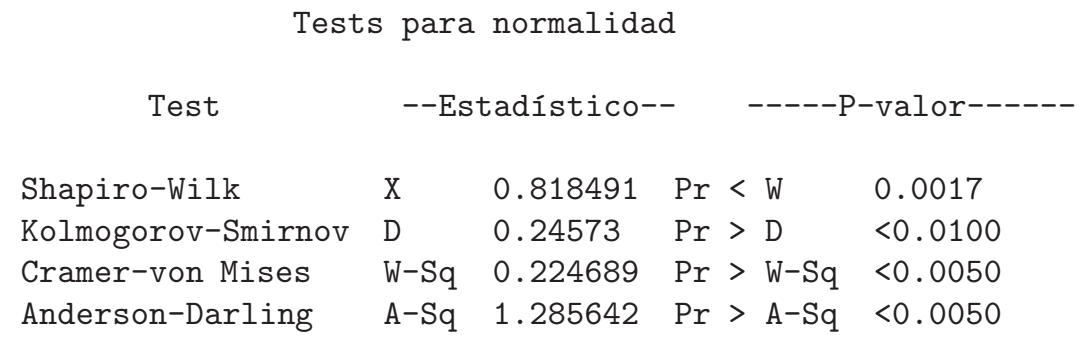

La siguiente es la salida de los valores calculados de las estadísticas $Q_{i}, T_{i}$ y el P-valor para las 20 observaciones.

\begin{tabular}{cccrrr} 
OBS & $\mathrm{Y}$ & $\mathrm{T}$ & \multicolumn{1}{c}{$\mathrm{QI}$} & \multicolumn{1}{c}{ PORCENT } & P_VALOR \\
1 & 38.91 & 2.735781 & 74.474782 & 49.867976 & 0.014081 \\
2 & 25.57 & 0.609178 & 4.31418 & 2.888760 & 0.550461 \\
3 & 26.71 & 0.111903 & 0.13287 & 0.088975 & 0.912210 \\
4 & 26.67 & 0.138609 & 0.21023 & 0.140770 & 0.891387 \\
5 & 23.94 & 0.821165 & 6.54848 & 4.384838 & 0.422918 \\
6 & 33.43 & 1.909870 & 39.819893 & 26.663219 & 0.073171 \\
7 & 26.07 & 0.010125 & 0.00099 & 0.000663 & 0.992039 \\
8 & 25.13 & 0.194368 & 0.37245 & 0.249396 & 0.848191 \\
9 & 24.32 & 0.355000 & 1.30181 & 0.871689 & 0.726955 \\
10 & 26.24 & 0.405438 & 1.83429 & 1.228236 & 0.690212 \\
11 & 25.27 & 0.107500 & 0.11859 & 0.079412 & 0.915650 \\
12 & 32.76 & 0.973090 & 8.88769 & 5.951158 & 0.344152 \\
13 & 28.81 & 0.376841 & 1.34048 & 0.897583 & 0.710953 \\
14 & 25.35 & 0.764101 & 5.66150 & 3.790915 & 0.455282 \\
15 & 26.93 & 0.894445 & 7.48149 & 5.009574 & 0.383572 \\
16 & 28.80 & 0.986754 & 9.79388 & 6.557942 & 0.337600 \\
17 & 29.74 & 0.125805 & 0.15485 & 0.103690 & 0.901361 \\
18 & 29.62 & 0.962537 & 9.77693 & 6.546588 & 0.349272 \\
19 & 30.28 & 0.123039 & 0.16328 & 0.109333 & 0.903519 \\
20 & 34.78 & 0.216738 & 0.69088 & 0.462611 & 0.830991
\end{tabular}

Las estadísticas máx $\left(Q_{i}\right)=74.4747, T_{i}=2.7357$ y el P-valor $=0.014081$ calculadas para el primer registro $\left(y, x_{1}, x_{2}\right)=(38.91,4.2,1.9)$, caracterizan esta observación como influyente según el criterio definido y el valor de $Q_{i}=$ 74.47 corresponde al valor en el cual se disminuye la $S C E$ si se ajusta el modelo después de eliminar este registro con una variación porcentual de $49.87 \%$. 
- En el segundo paso, se elimina la observación caracterizada como influyente y se repite el proceso para las 19 observaciones restantes. Resultado de ajustar el modelo después de eliminar el primer registro son: la $S C E=74.86$ en el nuevo modelo, el coeficiente de determinación aumenta $R^{2}=0.58$ y las pruebas de normalidad de los residuales mejoran. Las estadísticas y prueba de normalidad de los residuales y los valores de las estadísticas $Q_{i}, T_{i}$ para el modelo ajustado con las 19 observaciones son las siguientes.

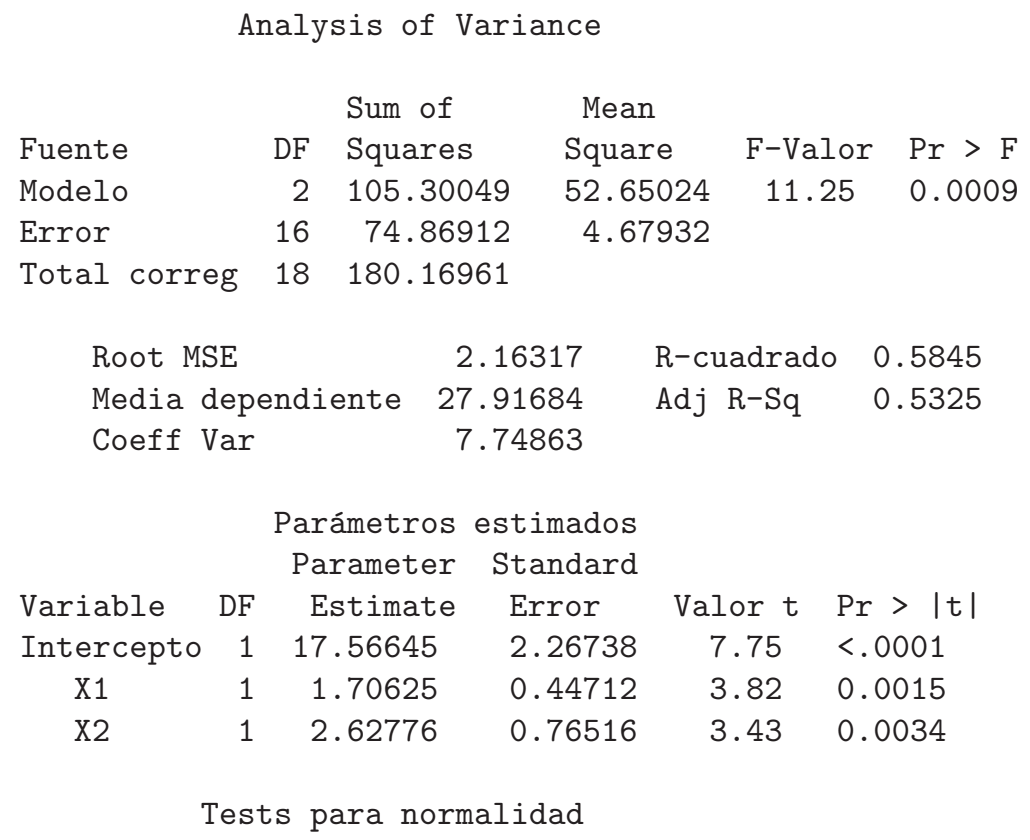

\begin{tabular}{lcrlr} 
Test & --Estadístico-- & \multicolumn{2}{l}{---- - } & \\
Shapiro-Wilk & X & 0.907531 & Pr $<$ W & 0.0667 \\
Kolmogorov-Smirnov & D & 0.117669 & Pr $>$ D & $>0.1500$ \\
Cramer-von Mises & W-Sq & 0.070528 & Pr $>$ W-Sq & $>0.2500$ \\
Anderson-Darling & A-Sq & 0.524632 & Pr $>$ A-Sq & 0.1636
\end{tabular}

\begin{tabular}{rrrrrl} 
OBS & $\mathrm{Y}$ & $\mathrm{T}$ & $\mathrm{QI}$ & PORCENT & \multicolumn{1}{l}{ P_VALOR } \\
1 & 25.57 & 1.01240 & 6.36358 & 8.49961 & 0.326411 \\
2 & 26.71 & 0.27872 & 0.43961 & 0.58718 & 0.784028 \\
3 & 26.67 & 0.33981 & 0.67421 & 0.90052 & 0.738417 \\
4 & 23.94 & 1.14589 & 6.79244 & 9.07241 & 0.268689 \\
5 & 33.43 & 2.50599 & 36.55217 & 48.82143 & 0.023390 \\
6 & 26.07 & 0.04544 & 0.01063 & 0.01420 & 0.964313 \\
7 & 25.13 & 0.18242 & 0.17484 & 0.23353 & 0.857542 \\
8 & 24.32 & 0.35019 & 0.67569 & 0.90250 & 0.730759 \\
9 & 26.24 & 0.74406 & 3.30005 & 4.40776 & 0.467624 \\
10 & 25.27 & 0.01009 & 0.00055 & 0.00074 & 0.992068
\end{tabular}




$\begin{array}{rrrrrr}11 & 32.76 & 1.62681 & 13.30828 & 17.77539 & 0.123306 \\ 12 & 28.81 & 0.76628 & 2.96488 & 3.96009 & 0.454663 \\ 13 & 25.35 & 0.74801 & 2.90799 & 3.88410 & 0.465305 \\ 14 & 26.93 & 0.90422 & 4.10095 & 5.47749 & 0.379291 \\ 15 & 28.80 & 0.96264 & 5.01969 & 6.70462 & 0.350049 \\ 16 & 29.74 & 0.28596 & 0.43253 & 0.57772 & 0.778575 \\ 17 & 29.62 & 0.77128 & 3.42119 & 4.56956 & 0.451781 \\ 18 & 30.28 & 0.42676 & 1.07576 & 1.43685 & 0.675237 \\ 19 & 34.78 & 0.28417 & 0.6559444 & 0.87612 & 0.779918\end{array}$

En el nuevo análisis de residuales en el ajuste del modelo después de eliminar la primera observación caracterizada como influyente, las estadísticas máx $\left(Q_{i}\right)=36.5521, T_{i}=2.5059$ y el $\mathrm{P}$-valor $=0.0233$ calculadas para el registro $\left(y, x_{1}, x_{2}\right)=(33.43,1.5,3)$, caracterizan esta observación como influyente según el criterio definido y el valor de $Q_{i}=36.5521$ corresponde al valor en el cual se disminuye la $S C E$ si se ajusta el modelo después de eliminar este registro con una variación porcentual de $48.82 \%$. El valor de la estadística $Q_{(i, j)}=111.0268=74.4747+36.5521$ es el valor máximo de variación de la $S C E$ al eliminar una pareja de observaciones, en este caso las observaciones $(38.91,4.2,1.9)$ y $(33.43,1.5,3)$.

- En el tercer paso, se elimina la segunda observación caracterizada como influyente y se repite el proceso para las 18 observaciones restantes. Resultado de ajustar el modelo después de eliminar este registro son: la $S C E=38.3169$ en el nuevo modelo, el coeficiente de determinación aumenta $R^{2}=0.7413$ y las pruebas de normalidad de los residuales mejoran. Las estadísticas y prueba de normalidad de los residuales y los valores de las estadísticas $Q_{i}, T_{i}$ para el modelo ajustado con las 18 observaciones son las siguientes.

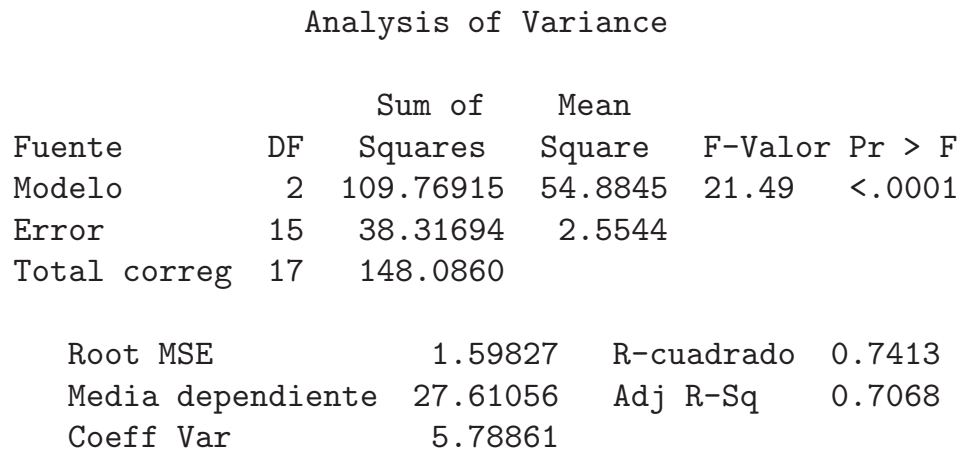


Parámetros estimados

\begin{tabular}{lrrrrr}
\multicolumn{1}{c}{ Parameter } & Standard & & \\
Variable & DF & Estimate & Error & Valor $t$ & Pr $>|t|$ \\
Intercepto & 1 & 17.70481 & 1.67566 & 10.57 & $<.0001$ \\
$\quad$ X1 & 1 & 2.00961 & 0.33995 & 5.91 & $<.0001$ \\
X2 & 1 & 1.98931 & 0.58999 & 3.37 & 0.0042
\end{tabular}

\begin{tabular}{|c|c|c|c|c|}
\hline Test & \multicolumn{2}{|c|}{-Estadístico-- } & \multicolumn{2}{|c|}{$-----P-v a l$ or------ } \\
\hline Shapiro-Wilk & $\mathrm{X}$ & 0.90989 & $\operatorname{Pr}<W$ & 0.0857 \\
\hline Kolmogorov-Smirnov & $\mathrm{D}$ & 0.14952 & $\operatorname{Pr}>\mathrm{D}$ & $>0.1500$ \\
\hline ramer-von Mises & W-Sq & 0.05900 & $\mathrm{Pr}>\mathrm{W}-\mathrm{Sq}$ & $>0.2500$ \\
\hline Anderson-Darling & $\mathrm{A}-\mathrm{Sq}$ & 0.49665 & $\mathrm{Pr}>\mathrm{A}-\mathrm{Sq}$ & 0.1942 \\
\hline
\end{tabular}

$\begin{array}{rrrrrr}\text { OBS } & \mathrm{Y} & \mathrm{T} & \mathrm{QI} & \text { PORCENT } & \text { P_VALOR } \\ 1 & 25.57 & 0.44624 & 0.73296 & 1.912889 & 0.661793 \\ 2 & 26.71 & 0.38895 & 0.49174 & 1.283374 & 0.702777 \\ 3 & 26.67 & 0.36519 & 0.45192 & 1.179435 & 0.720064 \\ 4 & 23.94 & 1.10031 & 3.47341 & 9.064970 & 0.288538 \\ 5 & 26.07 & 0.35429 & 0.35507 & 0.926684 & 0.728045 \\ 6 & 25.13 & 0.11190 & 0.03597 & 0.093879 & 0.912379 \\ 7 & 24.32 & 0.49678 & 0.74232 & 1.937338 & 0.626545 \\ 8 & 26.24 & 0.82644 & 2.22898 & 5.817224 & 0.421503 \\ 9 & 25.27 & 0.02814 & 0.00236 & 0.006172 & 0.977933 \\ 10 & 32.76 & 2.40763 & 15.96346 & 41.661643 & 0.029383 \\ 11 & 28.81 & 1.06022 & 3.09849 & 8.086500 & 0.305818 \\ 12 & 25.35 & 1.10716 & 3.48028 & 9.082895 & 0.285663 \\ 13 & 26.93 & 1.11686 & 3.41842 & 8.921444 & 0.281622 \\ 14 & 28.80 & 1.07213 & 3.41375 & 8.909262 & 0.300608 \\ 15 & 29.74 & 0.26026 & 0.19584 & 0.511109 & 0.798197 \\ 16 & 29.62 & 1.06583 & 3.56669 & 9.308396 & 0.303354 \\ 17 & 30.28 & 0.21711 & 0.15375 & 0.401270 & 0.831046 \\ 18 & 34.78 & 0.66524 & 1.98124 & 5.170681 & 0.515991\end{array}$

En el nuevo análisis de residuales en el ajuste del modelo después de eliminar la segunda observación caracterizada como influyente, las estadísticas máx $\left(Q_{i}\right)=15.9634 T_{i}=2.4076$ y el $\mathrm{P}$-valor $=0.0293$ calculadas para el registro $\left(y, x_{1}, x_{2}\right)=(32.763 .3,2.3)$, caracterizan esta observación como influyente según el criterio definido y el valor de $Q_{i}=15.9634$ corresponde al valor en el cual se disminuye la $S C E$ si se ajusta el modelo después de eliminar este registro con una variación porcentual de $41.66 \%$. El valor de la estadística $Q_{(i, j, k)}=126.9902=111.0268+15.9634$ es el valor máximo de variación de la $S C E$ al eliminar una terna de observaciones, en este caso las observaciones $(38.91,4.2,1.9),(33.43,1.5,3)$ y $(32.763 .3,2.3)$. 
- En el ultimo paso, se elimina la tercera observación $\left(y, x_{1}, x_{2}\right)=(32.763 .3,2.3)$ caracterizada como influyente y se repite el proceso para las 17 observaciones restantes. Las estadísticas y prueba de normalidad de los residuales y los valores de las estadísticas $Q_{i}, T_{i}$ para el modelo ajustado con las 17 observaciones son las siguientes.

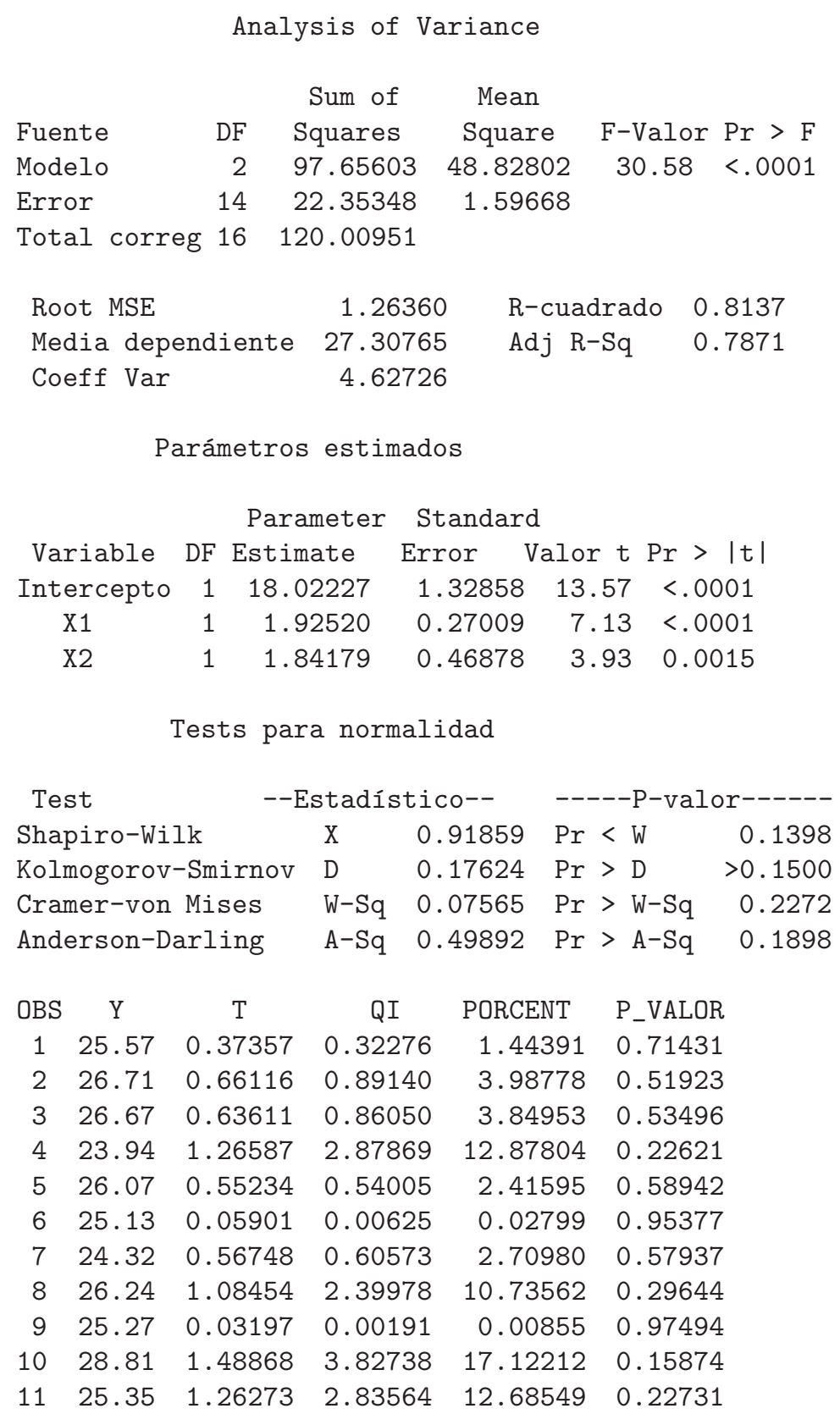

Comunicaciones en Estadística, diciembre 2010, Vol. 3, No. 2 


$\begin{array}{rrrrrr}12 & 26.93 & 1.19159 & 2.44503 & 10.93806 & 0.25322 \\ 13 & 28.80 & 1.04991 & 2.06883 & 9.25508 & 0.31154 \\ 14 & 29.74 & 0.55533 & 0.56054 & 2.50764 & 0.58743 \\ 15 & 29.62 & 1.03021 & 2.10907 & 9.43510 & 0.32037 \\ 16 & 30.28 & 0.50580 & 0.52518 & 2.34947 & 0.62086 \\ 17 & 34.78 & 1.28447 & 4.78126 & 21.38937 & 0.21982\end{array}$

En el ajuste del modelo sin las 3 observaciones caracterizadas como influyentes en los pasos previos, según el criterio de influencia ya no existen observaciones influyentes, la $S C E=22.35$, el coeficiente de determinación aumenta $R^{2}=0.81 .37$ y las pruebas de normalidad de los residuales ahora no proporcionan evidencia para rechazar la hipótesis de que los residuales tienen distribución normal.

\section{Conclusiones}

Es necesario al ajustar un modelo de regresión vía mínimos cuadrados, realizar metódicamente el análisis de residuales para detectar las observaciones influyentes en la estimación de la varianza residual. La experiencia acumulada al aplicar este procedimiento estadístico, muestra cómo en ocasiones un solo dato atípico o influyente aumenta significativamente el error en el modelo, lo cual disminuye la calidad de la estimación, y es necesario en el análisis de los datos establecer los diferentes escenarios para que el experto en el tema de interés visualice las diversas opciones que muestra el procedimiento estadístico en el modelamiento de los datos.

Claramente no es suficiente con caracterizar las observaciones influyentes para la estimación de la varianza residual. Se sabe que una observación influyente en este sentido, al ser eliminada genera, cambios en:

- La estimación de los parámetros, lo cual se puede observar con la estadística DFbeta.

- El coeficiente de determinación.

- La desviación estándar del estimador de cada parámetro y por tanto, en su significancia en el modelo.

- Las pruebas para validar los supuestos adoptados sobre los residuales.

Por todo lo anterior, se debe incluir como parte del modelamiento el estudio de estos escenarios en donde se explique y justifique la influencia global que un registro o grupo de registros tiene sobre el comportamiento de las estadísticas usualmente utilizadas para evaluar, valorar y validar el modelo ajustado o recta de regresión recomendada para su uso en producción. Agregar estos análisis al proceso de modelamiento, es importante para que el experto decida o seleccione el escenario o modelo que mejor interpreta el proceso en el cual se genera la información. 
Recibido: 20 de agosto de 2010 Aceptado: 24 de septiembre de 2010

\section{Referencias}

Draper, N. R. \& John, J. A. (1981), 'Influential observations and outliers in regression', Technometrics 23(1), $21-26$.

Jiménez, J. A. \& Rincón, L. F. (2000), 'Una generalización de la estadística dfbeta', Revista Colombiana de Estadística 23(1).

Morales, M. A. (2000), Estudio de algunas consecuencias derivadas de eliminar una observación influyente en modelos de regresión lineal múltiple, Trabajo de Grado, Especialización en Estadística, Universidad Nacional de Colombia.

Rincón, A. T. (1999), Propuesta para caracterizar observaciones influyentes en Modelos de Regresión Lineal Múltiple, Trabajo de Grado, Pregrado en Estadística, Universidad Nacional de Colombia.

Rincón, L. F. (2009), 'Un criterio que compara las estadísticas $q_{i}$ y $d f \beta_{j}(i)$ para el análisis de residuales en modelos de rango completo', Comunicaciones en Estadística 2(2), 139 - 146.

Searle, S. R. (1971), Linear Models, Wiley.

\section{Apéndice}

Finalmente se anexan los programas que se ejecutan en SAS o en $\mathrm{R}$ y que proporcionan el valor de las estadísticas objeto de estudio.

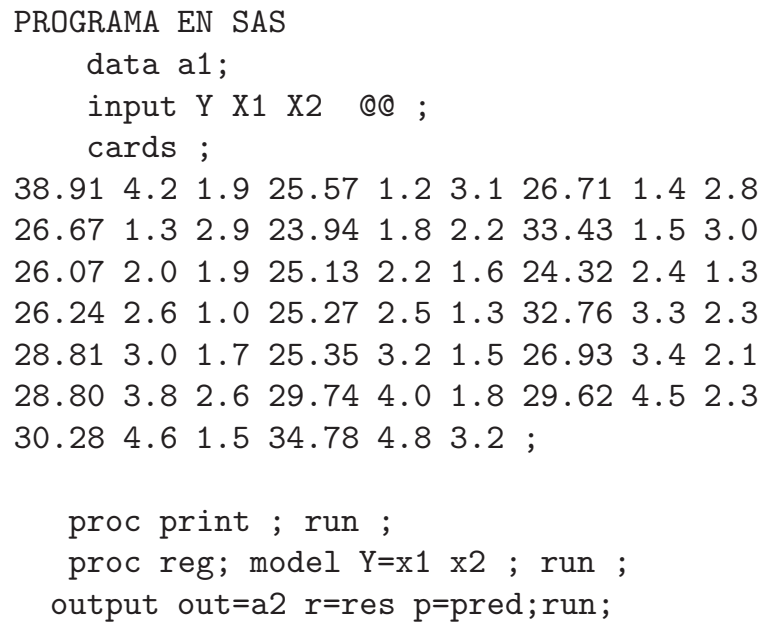




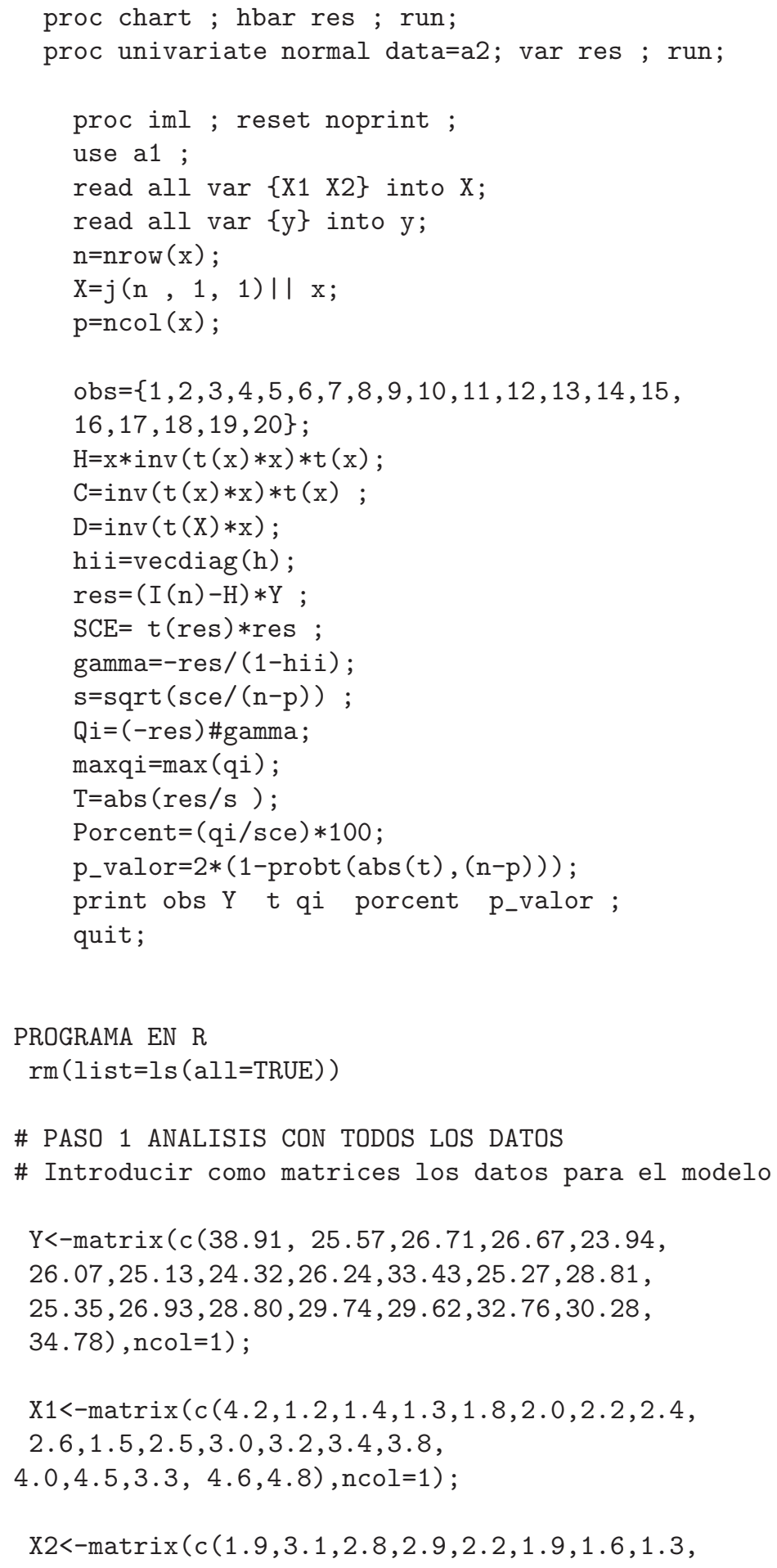




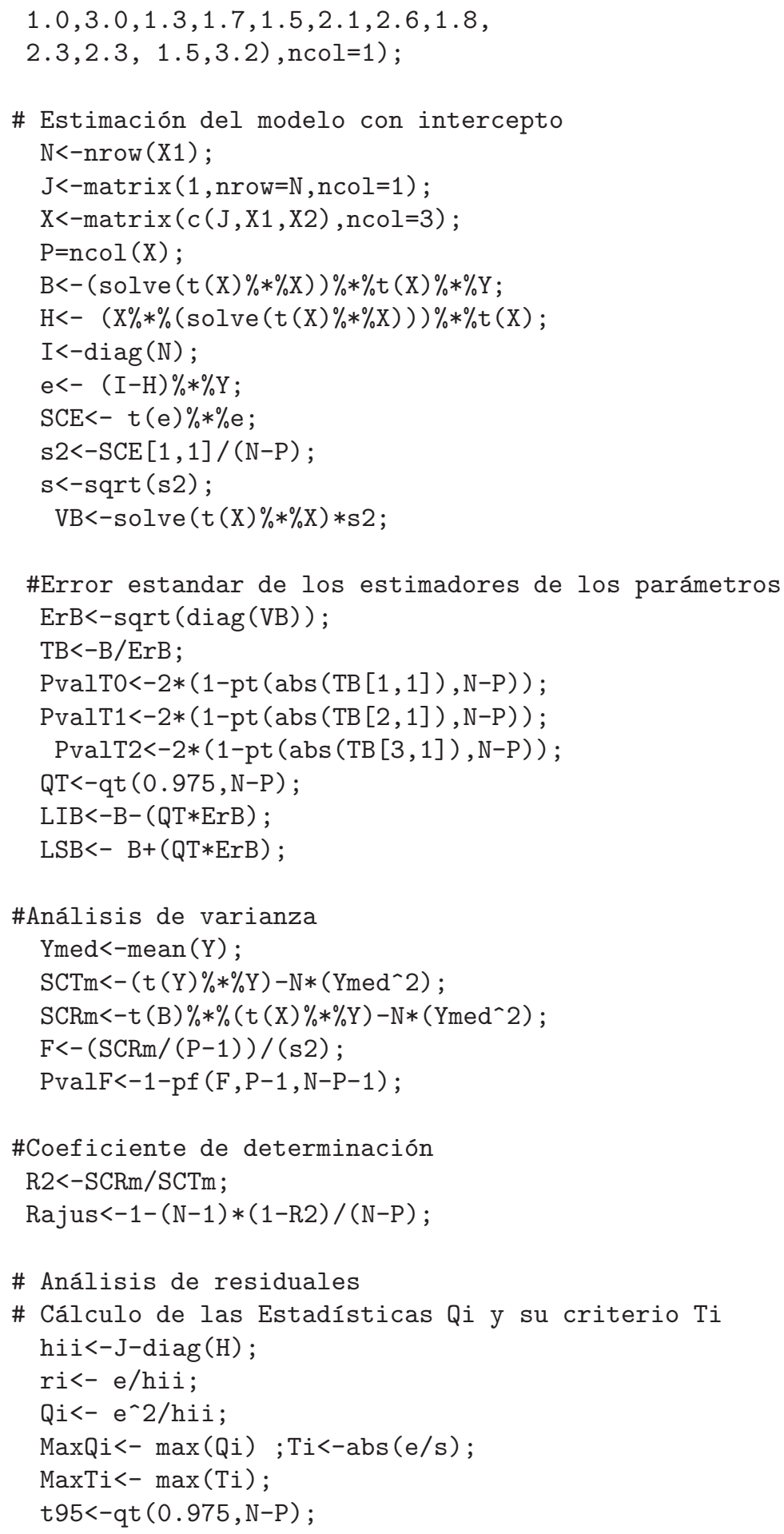




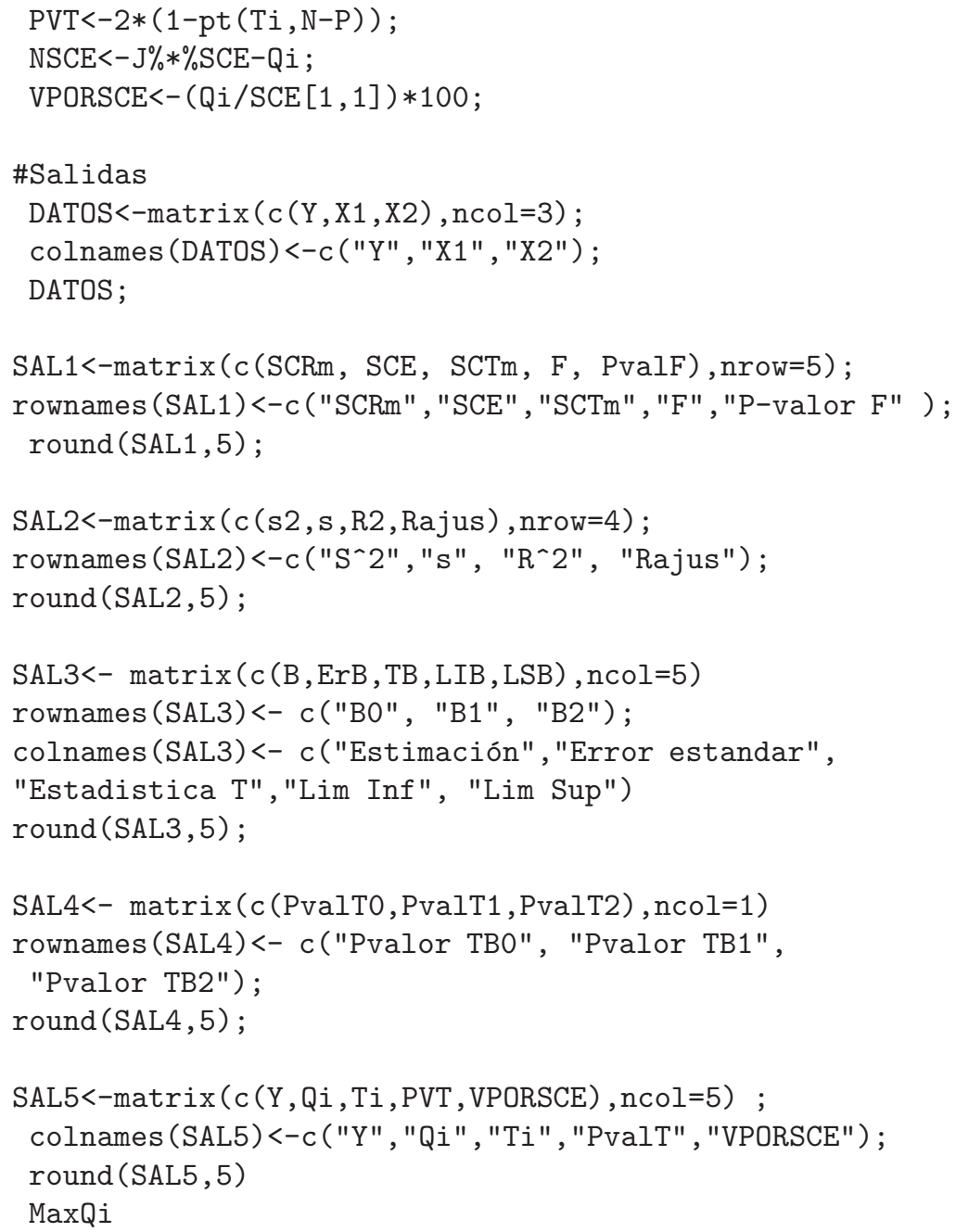

